\title{
Raised VEGF
}

\section{High sensitivity and specificity in the diagnosis of POEMS syndrome}

\author{
Morgane Pihan, MD, Stephen Keddie, MRCP, * Shirley D'Sa, FRCPath, Andrew J. Church, PhD, \\ Kwee L. Yong, PhD, Mary M. Reilly, FRCPI, and Michael P. Lunn, PhD
}

Neurol Neuroimmunol Neuroinflamm 2018;5:e486. doi:10.1212/NXI.0000000000000486

\section{Abstract}

\section{Objective}

To investigate the sensitivity and the specificity of serum vascular endothelial growth factor (sVEGF) for the diagnosis of polyneuropathy, organomegaly, endocrinopathy, M-protein, and skin changes (POEMS) syndrome in patients with a neuropathy (NP) and to identify confounding causes of raised vascular endothelial growth factor (VEGF) in this context to improve accuracy.

\section{Methods}

We studied the specificity and sensitivity of sVEGF for the diagnosis of POEMS syndrome in a cohort of 195 consecutive patients with an NP in serum samples from June 2009 to November 2013, including 27 untreated patients with POEMS syndrome. We then studied VEGF in other neuropathies and analyzed causes of elevated VEGF in a multiple logistic regression analysis in a larger cohort of 236 patients including 168 with a non-POEMS NP and 68 without NP.

\section{Results}

The sensitivity of elevated sVEGF for the diagnosis of POEMS was 100\%. Its specificity was 91\% in patients with an NP and $92 \%$ in patients with an NP and a paraproteinemia. sVEGF was much higher in POEMS before treatment. sVEGF was not significantly elevated in any nonPOEMS NP or hematologic disease group. Multiple logistic regression showed that anemia with low iron was a significant predictor for elevated sVEGF and that chronic obstructive pulmonary disease (COPD) and obstructive sleep apnoea-hypopnoea syndrome were significant predictors for very elevated sVEGF.

\section{Interpretation}

We confirmed the high sensitivity and specificity of an elevated VEGF for the diagnosis of POEMS. However, VEGF testing should be repeated, particularly after acute illnesses. Raised sVEGF should be interpreted with caution unless anemias with low iron, sleep apnea, COPD, cancers, vasculitis, and chronic inflammatory diseases are excluded.

\section{Classification of evidence}

This study provides class IV evidence that elevated sVEGF levels accurately identifies patients with POEMS syndrome.

\author{
Correspondence \\ Stephen Keddie \\ Stephen.keddie@nhs.net or \\ Dr. Lunn \\ michaellunn@nhs.net
}

MORE ONLINE

$\rightarrow$ Class of Evidence

Criteria for rating

therapeutic and diagnostic studies

NPub.org/coe

\footnotetext{
*Co-first author.

From the MRC Centre for Neuromuscular Diseases (M.P., S.K., M.M.R., M.P.L.), National Hospital for Neurology and Neurosurgery, Queen Square, London, UK; Cancer Division (S.D. K.L.Y.), University College London Hospitals NHS Foundation Trust, London, UK; and Department of Neuroimmunology (A.J.C., M.P.L.), Institute of Neurology, Queen Square, London, UK.

Funding information and disclosures are provided at the end of the article. Full disclosure form information provided by the authors is available with the full text of this article at Neurology.org/NN.

The Article Processing Charge was funded by the authors.

The statistical analyses were completed by Morgane Pihan, MRC Centre for Neuromuscular Diseases, National Hospital for Neurology and Neurosurgery, Queen Square, London, UK. 


\section{Glossary}

COPD = chronic obstructive pulmonary disease; HD = hematologic disease; Ig = immunoglobulin; IL-6 = interleukin 6; OSAHS = obstructive sleep apnoea-hypopnoea syndrome; NP = neuropathy; POEMS = polyneuropathy, organomegaly, endocrinopathy, M-protein, and skin changes; sVEGF = serum vascular endothelial growth factor; VEGF = vascular endothelial growth factor; $\mathbf{W M}=$ Waldenström macroglobulinemia.

Polyneuropathy, organomegaly, endocrinopathy, M-protein, and skin changes (POEMS) syndrome is a rare and severe multisystem disease driven by an underlying plasma cell dyscrasia. Treatments include surgical removal or irradiation of isolated plasmacytoma and, when the plasma cell dyscrasia is disseminated, chemotherapy with or without hematopoietic stem cell transplantation. ${ }^{1}$ Raised circulating vascular endothelial growth factor (VEGF) levels have been reported in patients with POEMS. ${ }^{2-4}$ Because this appears to be a distinct characteristic from other paraproteinemic neuropathies, the diagnostic criteria for POEMS were modified in 2007 to include circulating VEGF as a major criterion. ${ }^{5}$ POEMS syndrome is confirmed when the 2 mandatory criteria of a polyneuropathy and a monoclonal plasma cell proliferative disorder are present, in association with at least one of the other major criteria (Castleman disease, sclerotic bone lesion(s), and VEGF elevation), and with at least one of the minor diagnostic criteria. ${ }^{5}$ Levels of circulating VEGF are also probably useful in the follow-up of patients with POEMS syndrome as a biomarker of response to treatment. ${ }^{4,6}$ However, elevated VEGF has been described in diseases other than POEMS ${ }^{4,7}$ and patients with a neuropathy (NP) and a paraproteinemia, and failure to respect this could potentially lead to an incorrect diagnosis of POEMS syndrome.

This study documents the specificity (Sp) and sensitivity (Se) of serum VEGF (sVEGF) for the diagnosis of POEMS syndrome in a retrospective cohort of patients with an NP and identifying confounding factors of raised sVEGF in the investigation of neuropathies with paraproteinemia.

\section{Methods}

The primary research questions to address in this study were as follows:

1. What is the sensitivity and specificity of raised sVEGF for the diagnosis of POEMS syndrome compared with other causes of NP or non-POEMS hematologic disease (HD)?

2. What confounding factors cause raised sVEGF?

This retrospective study included consecutive patients attending peripheral nerve outpatient clinics at the National Hospital for Neurology and Neurosurgery who had an sVEGF tested as part of their clinical investigations from June 2009 to November 2013. Samples were sent immediately for processing in the Neuroimmunology Department of the Institute of Neurology, Queen Square, London, United Kingdom. Only patients with available clinical data were included in the study. We collected their age, sex, and pathologic conditions at the time of sVEGF testing from their medical records. The cohort included both POEMS and non-POEMS patients. Patients with POEMS syndrome were diagnosed according to internationally accepted criteria ${ }^{1}$; all had a polyneuropathy, and a monoclonal plasma cell disorder identified on serologic testing, bone marrow biopsy with or without plasmacytoma histology. Serial testing was requested in some because levels of VEGF were high and unexplained or lower than expected when POEMS was suspected. Because the purpose of this study was to identify and explain the causation of increased VEGF levels in non-POEMS patients, the highest values in all patients were selected.

sVEGF levels were measured by commercial ELISA according to the manufacturer's instructions (R\&D Systems, Minneapolis, MI). The upper limit of the normal range was determined as $90 \%$ fit of sera from 108 normal healthy blood donors and was $771 \mathrm{pg} / \mathrm{mL}$. Current practice in hematology uses a cutoff of $1,000 \mathrm{pg} / \mathrm{mL}$ as a more significant elevation of sVEGF. In this study, sVEGF was considered as "elevated" when $\geq 771 \mathrm{pg} / \mathrm{mL}$ and "very elevated" when $\geq 1,000 \mathrm{pg} / \mathrm{mL}$.

IBM SPSS Statistics 21 was used for statistical analysis. Nonparametric tests (Mann-Whitney $U$ and Kruskal-Wallis) were used to compare sVEGF levels between groups and subgroups of disease because sVEGF values were not normally distributed, and some groups or subgroups had small numbers of patients. The Spearman correlation test was used for correlations between sVEGF and age. Chi-square tests were used to compare the frequency of elevated and very elevated sVEGF in the different groups of diseases. Multiple logistic regression analyses were used to identify independent risks factors for elevated and very elevated sVEGF in the non-POEMS patient population.

\section{Standard protocol approvals, registrations, and patient consents}

Ethics committee approval was not required for this laboratory audit and clinical assay development. Studies of this kind are approved under The Royal College of Pathologists guidance. All patient information is fully anonymized.

\section{Data availability}

Any anonymized data not published within the article will be shared by request from a qualified investigator.

\section{Results}

A total of 206 patients with an NP had at least 1 test for sVEGF. There were 70 women $(34 \%)$ and 136 men $(66 \%)$ aged $58.4 \pm$ 
14.3 years (mean $\pm S D$, range $19-92$ years). Thirty-eight patients had clinically confirmed POEMS syndrome fulfilled by criteria other than increased VEGF levels, including 27 pretreatment and 11 posttreatment POEMS cases, and 168 patients had non-POEMS NP (table 1). Of the 11 treated patients with POEMS syndrome, 6 had received an autologous bone marrow transplant, 2 radiotherapy, 2 cyclophosphamide/ dexamethasone, and 1 lenalidomide/dexamethasone.

\section{sVEGF in patients with neuropathy}

sVEGF was significantly higher in the pretreatment POEMS group than in any of the other NP groups $(p<0.0001)$. It was also higher in the posttreatment POEMS, CIDP, and antiMAG NP groups than in the other noninflammatory NP groups ( $p=0.013, p=0.023$, and $p=0.009$ ), and it was higher in the posttreatment POEMS and anti-MAG NP groups than in the other inflammatory and paraproteinemic NP groups $(p=0.039$ and $p=0.025)$ (table 1 , figure 1$).$
All the pretreatment POEMS patients had very elevated sVEGF concentrations $(p<0.0001$ in comparison to other NP groups). The anti-MAG NP group had also a larger proportion of patients with elevated and very elevated sVEGF than CIDP, other inflammatory/paraproteinemic, and other noninflammatory NP groups $(p<0.05)$. Apart from the anti-MAG NP group ( $p=0.447$ vs posttreatment POEMS), more patients in the posttreatment POEMS group had an elevated sVEGF than in the other non-POEMS NP groups $(p<0.05)$, which was not found with very elevated sVEGF (table 1$)$.

sVEGF concentrations and the frequency of patients with raised sVEGF did not differ between the subgroups of the other inflammatory and paraproteinemic NPs $(p=$ $0.924)$, nor between the subgroups of other noninflammatory NPs $(p=0.392)$ (table e-1, links.lww.com/ NXI/A61).

Table 1 SVEGF concentrations and proportion of patients with raised SVEGF in the NP and hematologic disease groups ( $N=206$ patients)

\begin{tabular}{|c|c|c|c|c|c|c|c|}
\hline & \multirow[b]{3}{*}{ N patients (\%) } & \multicolumn{6}{|l|}{ sVEGF (pg/mL) } \\
\hline & & \multirow[b]{2}{*}{ Mean \pm SD } & \multirow{2}{*}{$\begin{array}{l}\text { Median [minimum; } \\
\text { maximum] }\end{array}$} & \multicolumn{2}{|c|}{$>771$} & \multicolumn{2}{|c|}{$>1,000$} \\
\hline & & & & $\mathbf{N}$ & $\% g p^{a}$ & $\mathbf{N}$ & $\%$ gp \\
\hline POEMS syndrome & $38(18.4 \%)$ & $2,741 \pm 2,652$ & $1,934[30 ; 10,956]$ & 32 & 84.2 & 28 & 73.7 \\
\hline Pretreatment & $27(13.1 \%)$ & $3,506 \pm 2,717$ & $2,030[1,010 ; 10,956]$ & 27 & 100 & 27 & 100 \\
\hline Posttreatment & $11(5.3 \%)$ & $864 \pm 1,170$ & $717[30 ; 4,247]$ & 5 & 45.5 & 1 & 9.1 \\
\hline Non-POEMS NP & $168(81.6 \%)$ & $364 \pm 324$ & $234[19 ; 2,616]$ & 15 & 8.9 & 11 & 6.5 \\
\hline CIDP & $51(24.8 \%)$ & $406 \pm 492$ & $271[41 ; 2,616]$ & 4 & 7.8 & 2 & 3.9 \\
\hline Anti-MAG NP ${ }^{b}$ & $19(9.2 \%)$ & $566 \pm 503$ & $370[59 ; 1,743]$ & 6 & 31.6 & 5 & 26.3 \\
\hline Vasculitic NP & $12(5.8 \%)$ & $450 \pm 601$ & $309[54 ; 2,261]$ & 1 & 8.3 & 1 & 8.3 \\
\hline Other inflammatory and paraproteinemic NPs & $33(16.0 \%)$ & $309 \pm 341$ & $209[34 ; 1,816]$ & 2 & 6.1 & 1 & 3.0 \\
\hline Other noninflammatory NPs & $55(26.7 \%)$ & $261 \pm 278$ & $153[19 ; 1,469]$ & 2 & 3.6 & 2 & 3.6 \\
\hline Non-POEMS hematologic disease & $88(42.7 \%)$ & $356 \pm 372$ & $234[28 ; 1,816]$ & 8 & 9.1 & 7 & 8.0 \\
\hline MGUS & $54(26.2 \%)$ & $376 \pm 374$ & $272[34 ; 1,743]$ & 5 & 9.3 & 4 & 7.4 \\
\hline Multiple myeloma & $18(8.7 \%)$ & $190 \pm 171$ & $105[28 ; 547]$ & 0 & 0 & 0 & 0 \\
\hline Other & $16(7.8 \%)$ & $475 \pm 475$ & $363[34 ; 1,816]$ & 3 & 18.8 & 3 & 18.8 \\
\hline Waldenstrom macroglobulinemia & $9(4.4 \%)$ & $517 \pm 349$ & $460[53 ; 1,069]$ & 2 & 22.2 & 2 & 22.2 \\
\hline Non-Hodgkin lymphoma ${ }^{c}$ & $4(1.9 \%)$ & $546 \pm 850$ & $168[34 ; 1,816]$ & 1 & 25 & 1 & 25 \\
\hline Other hematologic malignancies ${ }^{d}$ & $3(1.5 \%)$ & $254 \pm 182$ & $230[86 ; 447]$ & 0 & 0 & 0 & 0 \\
\hline No hematologic disease & $80(38.8 \%)$ & $373 \pm 477$ & $232[19 ; 2,616]$ & 7 & 8.8 & 4 & 5.0 \\
\hline Total & $206(100 \%)$ & $803 \pm 1,507$ & $311[19 ; 10,956]$ & 47 & 22.8 & 39 & 18.9 \\
\hline
\end{tabular}

Abbreviations: Anti-MAG = anti-myelin-associated glycoprotein; MGUS= monoclonal gammopathy of unknown significance; NP = neuropathy; POEMS = polyneuropathy, organomegaly, endocrinopathy, M-protein, and skin changes; sVEGF = serum vascular endothelial growth factor.

a Percentage of patients with increased VEGF levels in the disease group.

b Two patients with anti-MAG and vasculitic neuropathy.

${ }^{c}$ Follicular NHL $(n=1)$, Burkitt NHL $(n=1)$, and low-grade B-cell NHL $(n=2)$.

d Chronic lymphoid leukemia $(n=1)$, chronic myeloid leukemia $(n=1)$, and indolent T-cell large granular lymphocyte leukemia $(n=1)$. 


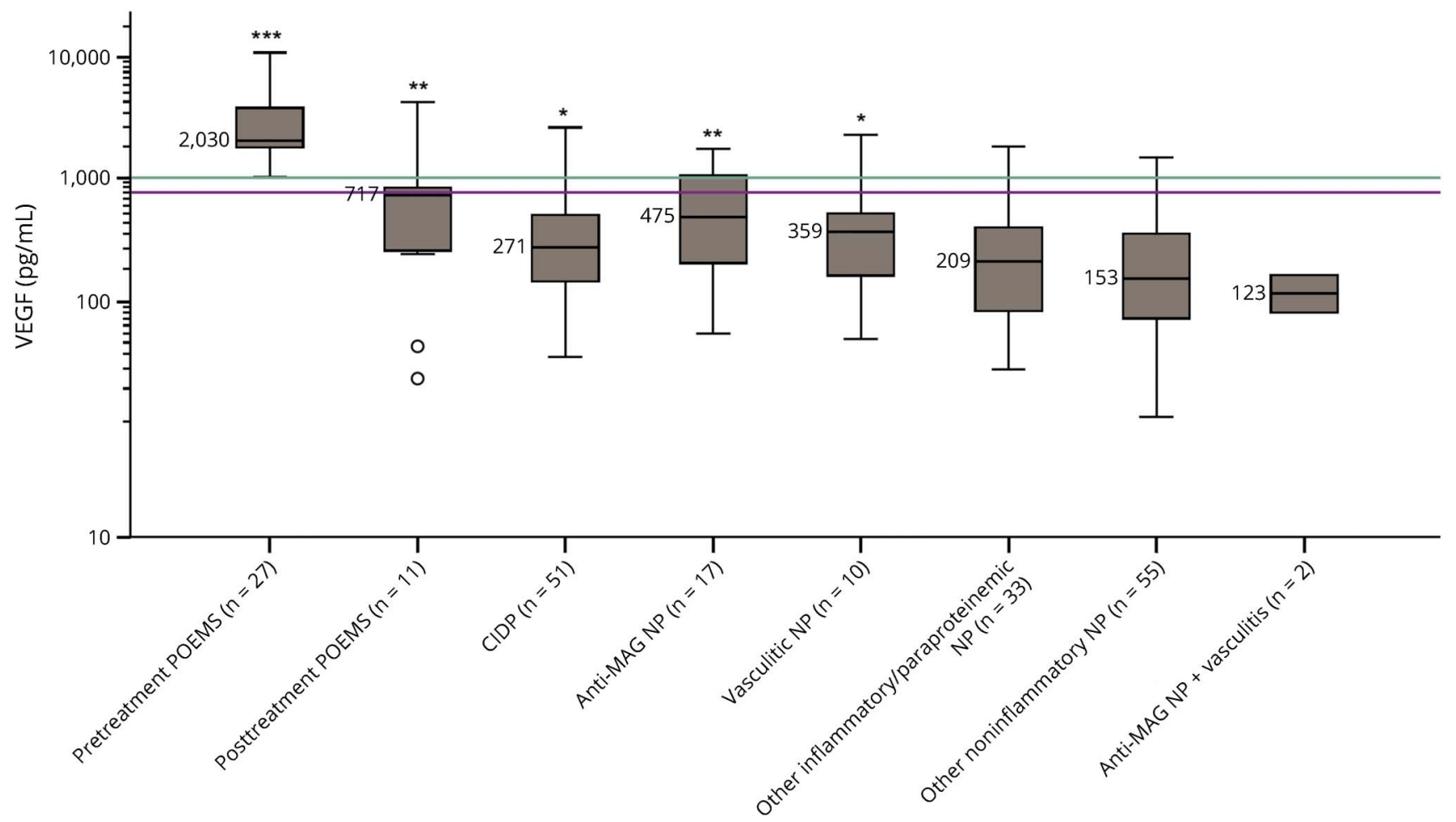

1,000 pg/mL (very elevated sVEGF); $771 \mathrm{pg} / \mathrm{mL}$ (elevated sVEGF). * $p<0.0001$ in comparison with each other group; ${ }^{\star} * p<0.05$ in comparison with other inflammatory/paraproteinemic NPs and other noninflammatory NPs; $* \star \star *<<0.05$ in comparison with other noninflammatory NPs. Anti-MAG = anti-myelinassociated glycoprotein; CIDP = chronic inflammatory demyelinating polyneuropathy; NP = neuropathy; POEMS = polyneuropathy, organomegaly, endocrinopathy, M-protein, and skin changes; VEGF = vascular endothelial growth factor.

\section{sVEGF in patients with hematologic disease}

Eighty-eight of 168 non-POEMS NP patients had a HD not always related to their NP (table 1). One patient with pretreatment POEMS syndrome had a nonsecretory monoclonal plasma cell disorder confirmed on bone marrow biopsy. sVEGF was significantly higher in the pretreatment POEMS than in the other HD groups $(p<0.01)$. Posttreatment POEMS had higher sVEGF than the multiple myeloma and no HD groups $(p<0.05)$ and similar sVEGF than the other HD groups. sVEGF concentrations were lower in the multiple myeloma group than in the pre- and post-treatment POEMS, MGUS, and Waldenström macroglobulinemia (WM) groups $(p<0.05)$, and it also tended to be lower than in the no HD group $(p=0.052)$.

\section{Sensitivity and specificity of sVEGF in POEMS syndrome}

For the Se-Sp study of sVEGF for the diagnosis of POEMS syndrome, posttreatment POEMS was excluded. This was studied in 195 patients with an NP (table 2). The area under the receiver operating characteristic curve (area under the curve (AUC) \pm standard error $[95 \% \mathrm{CI}(\mathrm{CI})]$ ) was $0.985 \pm$ 0.07 (0.971-0.998). In a subgroup of 109 patients with an NP and a paraproteinemia, i.e., who had the 2 mandatory major criteria for the diagnosis of POEMS syndrome ${ }^{5}$ (table 2), the AUC was $0.993 \pm 0.005$ (0.983-1.000).
Fifteen of 206 patients had a non-POEMS NP and a raised sVEGF, including 7 patients with a paraprotein (figure 2, table e-2, links.lww.com/NXI/A61). The mean \pm SD number of comorbidities per patient was $2.9 \pm 1.5$ (median 3.0, range 1-7). The number of comorbidities was not correlated with sVEGF concentrations $\left(r_{\mathrm{s}}=-0.09, p=0.246\right)$. There was no sex $(p=0.845)$ or age $\left(r_{\mathrm{s}}=-0.035, p=0.653\right)$ effect on sVEGF concentrations.

To further explain sVEGF elevation in those 15 patients with a non-POEMS NP and a raised sVEGF, all other comorbidities at the time of sVEGF testing were explored as potential confounding factors. Those analyses did not include patients with POEMS and thus explored 168 patients. Comorbidities were anemia $(n=20)$, diabetes $(n=18)$, chronic inflammatory disease $(n=12)$, ischemic disease (peripheral and coronary arterial diseases and stroke) $(\mathrm{n}=11)$, solid cancer $(\mathrm{n}=5)$, hypoxemic disease (chronic obstructive pulmonary disease [COPD], and obstructive sleep apnoea-hypopnoea syndrome [OSAHS]) $(\mathrm{n}=$ $10)$, hypertension $(n=47)$, other neurologic diseases $(n=14)$, other endocrinopathy $(n=13)$, benign tumurs $(n=11)$, dyslipidemia $(n=11)$, asthma $(n=9)$, nonischemic heart disease ( $n$ $=8)$, osteoporosis $(\mathrm{n}=8)$, chronic kidney disease $(\mathrm{n}=5)$, and miscellaneous conditions $(\mathrm{n}=29)$.

Anemia was defined as hemoglobin $\leq 12 \mathrm{~g} / \mathrm{dL}$ ( $\mathrm{n}=20 / 168$; $11.9 \%)$. There was no significant difference in sVEGF 
Table 2 Sensitivity and specificity of SVEGF for the diagnosis of POEMS syndrome in patients with an NP

\begin{tabular}{|c|c|c|c|c|c|}
\hline & sVEGF $(\mathrm{pg} / \mathrm{mL})$ & Se & Sp & NPV & PPV \\
\hline \multirow[t]{2}{*}{ NP $(N=195)$} & $>771$ & $100 \%$ & $91 \%$ & $100 \%$ & $64 \%$ \\
\hline & $>1,000$ & $100 \%$ & $93 \%$ & $100 \%$ & $71 \%$ \\
\hline \multirow{2}{*}{ NP and paraproteinemia $(\mathrm{N}=109)$} & $>771$ & $100 \%$ & $92 \%$ & $100 \%$ & $79 \%$ \\
\hline & $>1,000$ & $100 \%$ & $93 \%$ & $100 \%$ & $81 \%$ \\
\hline
\end{tabular}

Abbreviations: NP = neuropathy; NPV = negative predictive value; POEMS = polyneuropathy, organomegaly, endocrinopathy, M-protein, and skin changes; $\mathrm{PPV}=$ positive predictive value; $\mathrm{Se}=$ sensitivity; $\mathrm{Sp}=$ specificity; $\mathrm{SVEGF}=$ serum vascular endothelial growth factor.

NP: posttreatment POEMS excluded $(n=11)$; sVEGF > $771 \mathrm{pg} / \mathrm{mL}$ : elevated sVEGF; sVEGF >1,000 pg/mL: very elevated sVEGF.

concentrations in patients with anemia (median [min; max]: $331[28 ; 2,616] \mathrm{pg} / \mathrm{mL})$ or without anemia $(229[19 ; 2,443])$ $(p=0.428)$. However, when subgroups of anemia were defined according to their serum iron concentrations, there was a significant difference $(p=0.017)$ : sVEGF concentrations were significantly elevated in patients with anemia and low serum iron $(789[320 ; 2,616] \mathrm{pg} / \mathrm{mL}, \mathrm{n}=5)$ in comparison to patients without anemia $(229[19 ; 2,443] \mathrm{pg} / \mathrm{mL}, \mathrm{n}=148)$ $(p=0.005)$ and with anemia and normal serum iron (175 [28; $1,816] \mathrm{pg} / \mathrm{mL}, \mathrm{n}=15)(p=0.029)$. Of the 5 patients with anemia and low serum iron, 3 had CIDP, one an anti-MAG
NP, and 1 vasculitic NP. The clinical data of these 5 cases are summarized in table e-4, links.lww.com/NXI/A61. sVEGF concentrations did not differ in the low serum iron subgroup whether the iron profile showed an iron deficiency $(n=2)$ or a chronic inflammation pattern $(\mathrm{n}=3)$. Ten $(6 \%)$ patients had a hypoxemic disease, 4 with COPD, and 6 with OSAHS. Although the mean sVEGF concentrations were not significantly different between patients with $(291[69 ; 2,443]$ $\mathrm{pg} / \mathrm{mL})$ and without hypoxemic disease $(229[19 ; 1,616])$ $(p=0.400)$, the proportion of patients with a very elevated sVEGF was higher in patients with OSAHS representing 2/10

Figure 2 Distribution of serum vascular endothelial growth factor (sVEGF) in different neuropathy groups

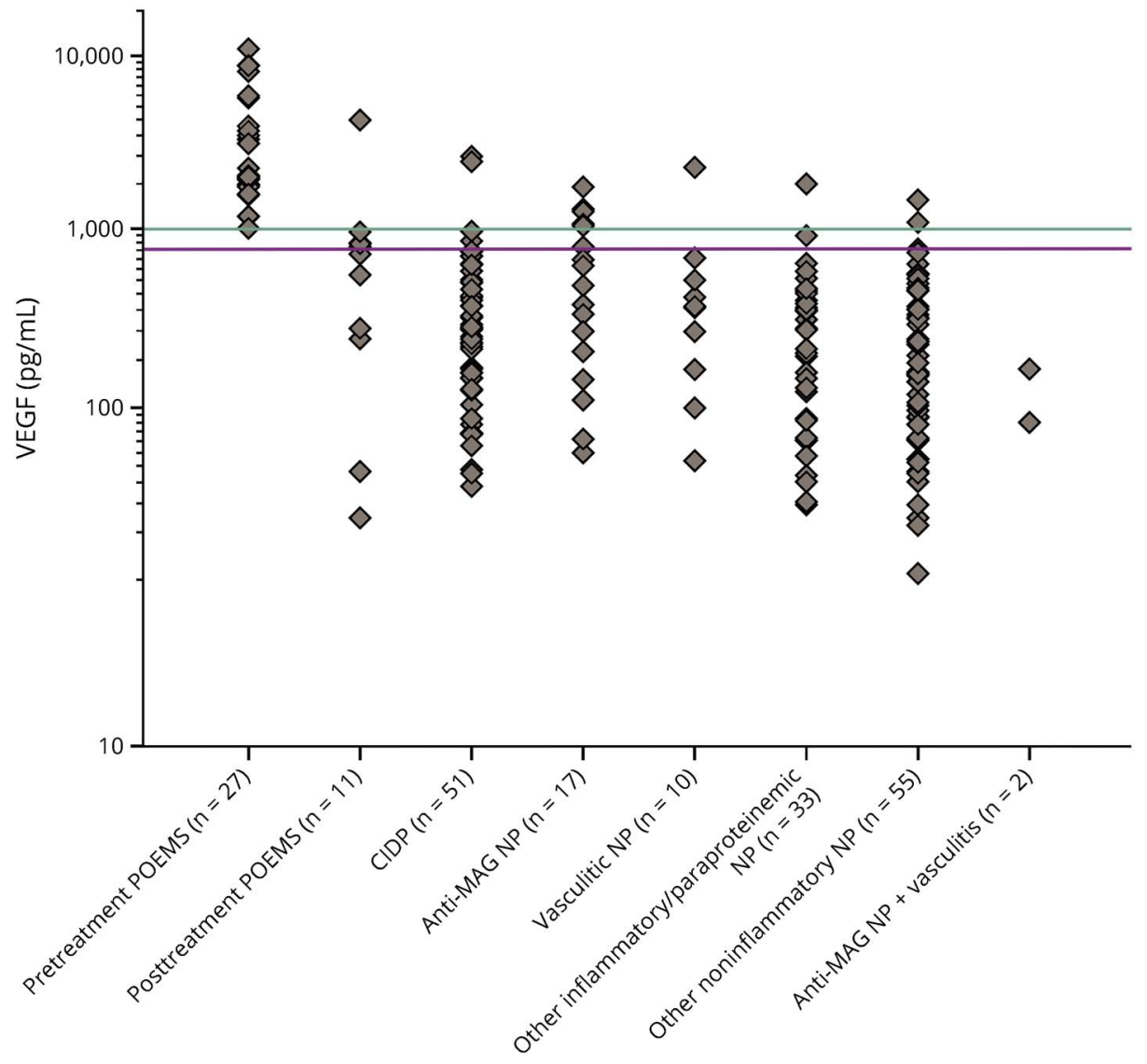

1,000 pg/mL (very elevated sVEGF); 771 pg/ $\mathrm{mL}$ (elevated sVEGF). Anti-MAG = anti-myelinassociated glycoprotein; CIDP = chronic inflammatory demyelinating polyneuropathy; $\mathrm{NP}$ = neuropathy; POEMS = polyneuropathy, organomegaly, endocrinopathy, M-protein, and skin changes; VEGF = vascular endothelial growth factor. 
(20.0\%) than in patients without a hypoxemic disease (9/158, $5.7 \%)(p=0.023)$. All 4 COPD patients had a stable disease without any recent exacerbation. Two of 6 patients with OSAHS were untreated at the time of testing and had a particularly high sVEGF concentration (1,043 and 2,443 pg/ $\mathrm{mL}$ ). No other comorbidities were associated with raised sVEGF concentration.

Because patients had multiple comorbidities, we explored risk factors for raised sVEGF with multiple logistic regression analyses. As some disease groups had small numbers of patients, we included 68 consecutive patients without an NP for whom sVEGF testing was requested between June 2009 and November 2013 and for whom clinical data were available. Thus, a total of 236 patients were analyzed. Twentythree of 236 patients (9.7\%) had an elevated sVEGF (8 without an NP), and 13/236 (5.5\%) had a very elevated sVEGF ( 2 without a NP). In addition to the non-POEMS NP and the HDs groups, the variables assessed for their predictive value are set out in table e-3, links.lww.com/NXI/A61, the most common of which were hypertension $(n=59)$, diabetes $(n=23)$, other endocrinopathies $(n=20)$, anemia with normal serum iron $(\mathrm{n}=32)$ and with low serum iron $(\mathrm{n}=6)$, other neurologic diseases $(n=26)$, and chronic inflammatory diseases $(n=23)$. The first model assessed the risk of any comorbidity for an "elevated" sVEGF. Anemia with low iron was the only significant predictor with an OR of 15.4 (95\% CI $1.3-187.8[p=0.032]$ ) (figure 3). In addition, there was a trend for COPD or OSAHS (OR $=7.9,95 \%$ CI 0.8-81.0, $p=$
$0.081)$ and anti-MAG NP ( OR $=8.6,95 \%$ CI 0.98-75.6, $p=0.052$ ) to be predictive of elevated sVEGF. The second model assessed the risk of having a "very elevated" sVEGF. Here, OSAHS or COPD was the only significant predictive factor $(\mathrm{OR}=41.7,95 \% \mathrm{CI} 1.4-1,226, p=0.031)$ (figure 3 ).

\section{Discussion}

This retrospective study confirms the high specificity and sensitivity of VEGF for the diagnosis of POEMS syndrome, especially when it is raised in the presence of an NP and a paraproteinemia. Similar findings using plasma VEGF in a cohort of 105 patients have also been demonstrated. ${ }^{4}$ By selecting patients with an NP, i.e., a population with a clinical presentation more such as POEMS syndrome and thus more likely to be tested, our study found a higher $\mathrm{Sp}$ and Se. This highlights the advantages of testing in a targeted population. A larger number of patients may also contribute to the accuracy of $\mathrm{Sp}$ and $\mathrm{Se}$. The use of plasma vs sVEGF is discussed. Higher concentrations of sVEGF reflect the release of VEGF from platelets during aggregation, and this could lead to a lack of reliability of sVEGF depending on collection and processing procedures. ${ }^{1}$ However, variations of plasma VEGF can also occur depending on those procedures, and standardization is needed for both plasma and serum testing. ${ }^{8}$ sVEGF might better reflect the VEGF overproduction in pathologic conditions and should probably be interpreted together with the platelet count. ${ }^{9}$ Timing of blood collection to processing was not standardized in this retrospective study, which could affect

Figure 3 SVEGF concentrations in anemia and hypoxemic diseases
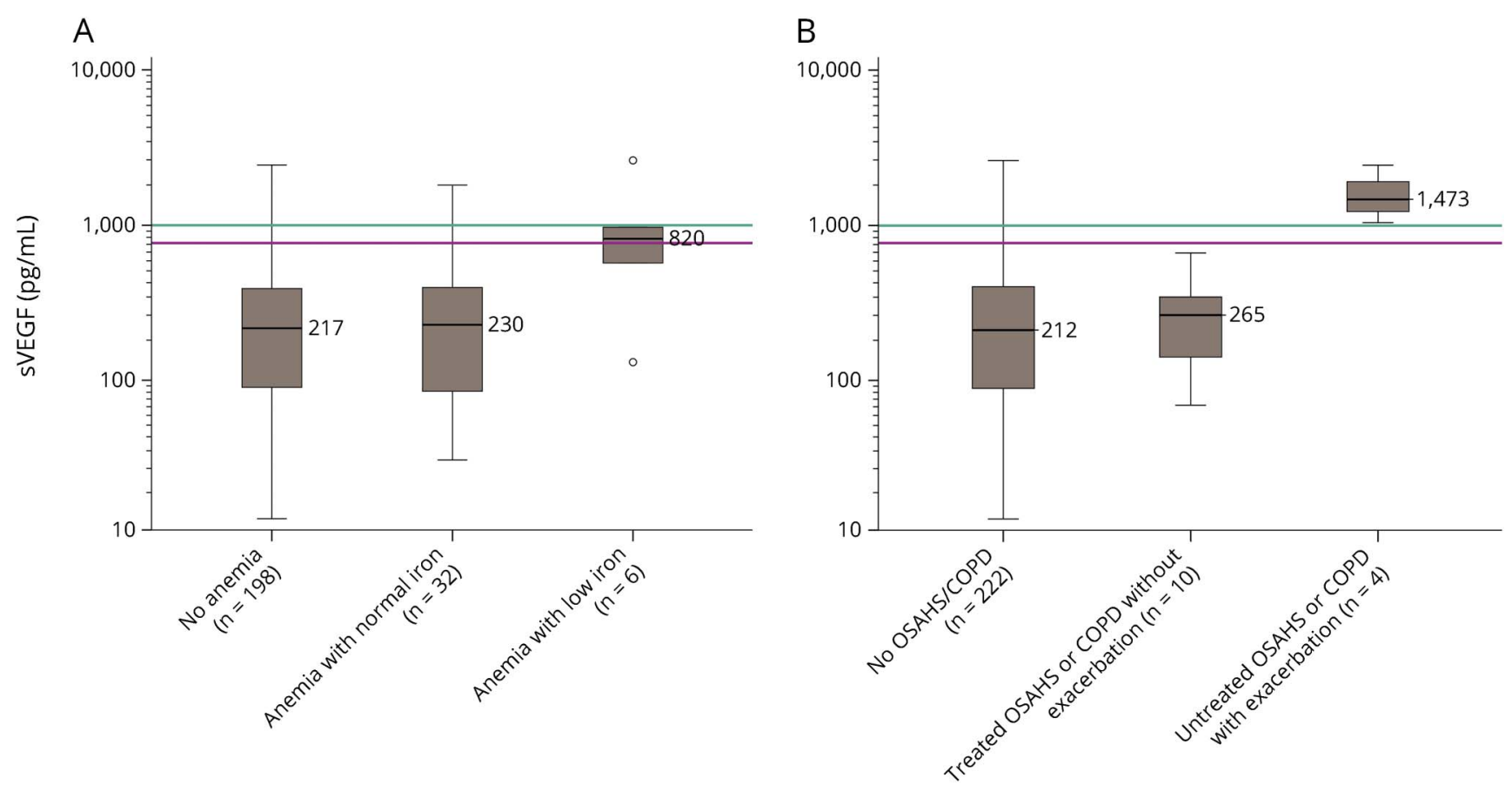

(A) Serum VEGF in anemia with and without iron deficiency. (B) Serum VEGF in hypoxemic diseases. 1,000 pg/mL (very elevated sVEGF); $771 \mathrm{pg} / \mathrm{mL}$ (elevated SVEGF). COPD = chronic obstructive pulmonary disease; OSAHS = obstructive sleep apnoea-hypopnea syndrome; sVEGF = serum vascular endothelial growth factor. 
the recorded sVEGF level. In another published cohort of 161 patients with an NP, lower specificity and positive predictive values were found, but 29 patients with amyotrophic lateral sclerosis were included. ${ }^{10}$ The proportions of patients with elevated sVEGF in the anti-myelin-associated glycoprotein (anti-MAG) NP, multifocal motor neuropathy (MMN), and other noninflammatory NP groups are similar to other studies. ${ }^{4,10}$ Higher sVEGF levels in patients with chronic inflammatory demyelinating polyradiculoneuropathy (CIDP) and Guillain-Barré Syndrome were not replicated in this study. This discrepancy might be at least partially explained by confounding comorbidities not explored in that study. In our study, none of the NP groups was found to be an independent predictive factor for raised sVEGF. However, comparative tests and the multiple logistic regression analysis support a raised sVEGF in some anti-MAG NPs, which requires confirmation by other studies. A raised sVEGF in this demyelinating NP associated with a paraproteinemia might lead to a type I error. However, the clinical presentation, progression, and the associated $\mathrm{HD}$ are so different that this misdiagnosis is unlikely. Anti-MAG NP is only associated with an immunoglobulin ( $\mathrm{Ig}$ ) M paraprotein usually with a kappa light chain; it is incredibly rare to have an IgM paraprotein associated with POEMS syndrome (2\%, of cases, compared with IgA $[52 \%]$ or $\operatorname{IgG}[42 \%]^{11}$ ). Finding an increased VEGF level, coupled with polyneuropathy and a non-IgM monoclonal paraprotein, especially with a lambda light chain, should be considered highly likely to be POEMS syndrome.

As expected, all pretreatment POEMS patients had very elevated sVEGF, significantly higher than in the other NP and HD groups. We did not look systematically at longitudinal sVEGF progression after treatment. However, the mean sVEGF was much lower in the posttreatment POEMS group, in line with previous studies that showed a VEGF decrease in response to treatment. ${ }^{3,4,6}$

No independent $\mathrm{HD}$ was associated with an elevated or very elevated VEGF in the absence of POEMS. Angiogenesis factors are involved in the pathogenesis of most of the hematologic malignancies, and raised circulating VEGF levels have been described in chronic myeloid leukemia, chronic myelomonocytic leukemia, chronic lymphocytic leukemia, and lymphoma, often in correlation with disease activity. ${ }^{12-14}$ The VEGF level has previously been found elevated in multiple myeloma (MM), ${ }^{15}$ but our study found lower sVEGF concentrations in this group in comparison to other $\mathrm{HD}$ groups and to patients with no $\mathrm{HD}$; this finding might be due to a large proportion $(16 / 18)$ of treated patients in our cohort. ${ }^{15}$ Increased VEGF levels has also been described in WM in 1 study. ${ }^{16}$ However, even if VEGF was higher in WM than in other HD groups, this did not reach significance here, and WM was not identified as a predictor of raised sVEGF in the multiple logistic regression models.

Anemia with low serum iron is a risk factor for elevated sVEGF. The small number of patients in this subgroup results in a wide $\mathrm{CI}$, but as anemia can lead to tissue hypoxia and stimulate VEGF secretion via hypoxia-inducible factor (HIF)-1-alpha stabilization, it is a plausible cause of raised circulating VEGF. ${ }^{7,17} \mathrm{We}$ could observe a significant decrease in sVEGF concentrations from 2,616 to $800 \mathrm{pg} / \mathrm{mL}$ after iron supplementation in a CIDP patient with iron deficiency anemia (patient 81 , table e-2, links. lww.com/NXI/A61). The association between increased VEGF levels and low hemoglobin levels has mainly been studied in patients with various cancers, and contradictory results were found. ${ }^{18,19}$ In our cohort, anemia on its own was not found to increase sVEGF levels, but it only became significant when associated with low serum iron. The effect of cellular iron deficiency on VEGF production has been demonstrated in breast cancer, supporting our results. This demonstrates in vitro that intracellular iron deficiency, independent from hypoxia, stabilizes HIF-1-alpha, which increases the production of VEGF. ${ }^{20}$ The upregulation of circulating VEGF secondary to iron deficiency has then been demonstrated in mouse studies. ${ }^{21}$

COPD and OSAHS are very significant risk factors for very elevated sVEGF. The multiple logistic regression model included 7 patients with a COPD and 7 with an OSAHS. The 4 patients with raised sVEGF had either untreated sleep apnea $(\mathrm{n}=2)$ or exacerbations of COPD $(\mathrm{n}=2)$ (figure 3$)$. Increased VEGF levels has been described in patients with mild to very severe COPD. ${ }^{22,23}$ Supporting our results, increased VEGF levels has been demonstrated in COPD exacerbations, correlated with systemic inflammatory markers, in comparison to stable COPD and healthy subjects, which has been also correlated inversely to arterial oxygenation. ${ }^{22,23}$ In OSAHS, previous studies found a raised circulating VEGF in untreated patients compared with treated patients and healthy controls, and this elevation was correlated with the nocturnal hypoxia and the apnea-hypopnea index. ${ }^{24,25}$

Contrary to our results, we did not find increased VEGF levels in a number of comorbidities that were possibly expected. VEGF is described as being raised in vasculitis, ${ }^{4,26}$ but this was not found in our study. However, our vasculitic neuropathies were mostly limited to the peripheral nervous system with no systemic involvement or associated inflammatory autoimmune disease. In connective tissue diseases, VEGF is associated with raised inflammatory cytokines and/or disease activity, ${ }^{27-29}$ and very high circulating VEGF levels have been found in patients with most active connective tissue diseases. ${ }^{27}$ In pulmonary infection, circulating VEGF can be elevated, ${ }^{30,31}$ explaining raised sVEGF in 2 cases of our cohort, 1 with pneumonia (patient 233) and another with organizing pneumonitis (patient 142). Both of them had very elevated sVEGF, which decreased after treatment. Solid cancers have been associated with increased VEGF levels. ${ }^{7,32}$ VEGF levels correlate with serum interleukin 6 (IL-6) (interesting in the context of IL-6 drive in POEMS, see below) and other cytokines, anemia, advanced cancer stage, and poor prognosis. ${ }^{18,33-40}$

Fifteen of 168 non-POEMS NP patients had a raised sVEGF, which could be explained by an identified comorbidity as above: anemia with low serum iron $(3 / 15$, patients 81,117 , 
and 176), COPD or sleep apnea (2/15, patients 145 and 238), connective tissue disease $(3 / 15$, patients 61,100 , and 44$)$, pulmonary infection (2/15, patients 142 and 233$)$, and lowgrade non-Hodgkin lymphoma (NHL) (1/15, patient 114) (table e-2, links.lww.com/NXI/A61). Retrospective data collection did not identify a potential cause of raised sVEGF in 4/15 patients including 3 patients with anti-MAG NP requiring further study.

VEGF upregulation by HIF-1-alpha and inflammatory cytokines is shared by all these conditions. In the pathogenesis of POEMS syndrome, VEGF is believed to be a key cytokine. It is produced in all tissues. Its receptors are found mainly on endothelial cells but also on hematopoietic stem cells, megakaryocytes, monocytes, chondrocytes, and others. Its neoangiogenesis and vascular permeability functions are likely responsible for some of the clinical features of POEMS, such as edema, pleural effusion, ascites, papilledema, and pulmonary hypertension. Increased endoneurial microvascular permeability is thought to contribute to blood-nerve barrier dysfunction, allowing ingress of toxic agents that cause nerve damage. ${ }^{41}$ Its osteogenesis function may be involved in sclerotic bone lesion formation. Thrombocytosis and polycythemia cause microvascular thrombosis and microvascular neoangiogenesis, inducing hypoxia and stabilizing HIF-1alpha resulting in an upregulation of VEGF. High VEGF is also sustained by inflammatory cytokines IL-1 beta, tumor necrosis factor (TNF) alpha, and IL-6. ${ }^{5,7,17,32}$

This retrospective study confirmed the high Se and Sp of sVEGF as a useful biomarker for POEMS syndrome diagnosis in patients with an NP, especially when associated with a paraproteinemia. In this context, only few important confounding comorbidities were identified, which should be considered during diagnosis. When clinical features are likely those of POEMS syndrome (a rapidly progressive demyelinating sensorimotor distal polyneuropathy with lambda light chain paraproteinemia), high VEGF is a very useful diagnostic biomarker for the diagnosis of POEMS syndrome, and the other clinical and biological features of POEMS syndrome should be systematically sought. On the contrary, when an increased VEGF level is found without a paraprotein and an appropriate NP, testing should be repeated, particularly after acute illnesses, and anemias with low iron, cancers, hematologic malignancies, OSAHS, COPD, vasculitis, and chronic inflammatory diseases should be excluded before using VEGF confidently for a POEMS diagnosis. sVEGF can be sent for analysis to the Neuroimmunology Department of the Institute of Neurology, Queen Square, London, United Kingdom.

\section{Author contributions}

M. Pihan: drafting/revising the manuscript, analysis or interpretation of data, and statistical analysis. S. Keddie: drafting/revising the manuscript, study concept or design, analysis or interpretation of data, acquisition of data, and statistical analysis. S.P. D'Sa: data acquisition, drafting/ revising the manuscript, study concept or design, analysis or interpretation of data, and acquisition of data. A.J. Church: data acquisition, acquisition of data, statistical analysis, and study supervision. K.L. Yong: data acquisition, drafting/ revising the manuscript, analysis or interpretation of data, and acquisition of data. M.M. Reilly: drafting/revising the manuscript and study concept or design. M.P. Lunn: data acquisition, drafting/revising the manuscript, study concept or design, analysis or interpretation of data, acquisition of data, statistical analysis, and study supervision.

\section{Study funding}

No targeted funding reported.

\section{Disclosure}

M. Pihan reports no disclosures. S. Keddie is a clinical research fellow based at the CSF and neuroimmunology laboratory, National Hospital for Neurology and Neurosurgeryalthough he does not directly process samples, this laboratory receives tests from across the United Kingdom to test VEGF, hence the purpose of investigational research into the utility of such a test-and receives research support from the Association of British Neurologists and Guarantors of Brain. S.P. D'Sa served on the speakers' bureau of Janssen and Amgen and received research support from Janssen. A.J. Church and K.L. Yong report no disclosures. M.M. Reilly served on the editorial boards of Brain, Neuromuscular Disorders, and JNNP; consulted for Servier, Acceleron, Alnylam, Ionis, and MyoTherix; and received research support from the NIH, MDA, MRC, Wellcome Trust, UCL CBRC, Ipsen Training Grant, NIHR, Muscular Dystrophy Campaign, and UCLH/UCL/ CRDC. M.P. Lunn served on the scientific advisory boards of Baxter, CSL Behring, and Grifols; received travel funding and/or speaker honoraria from CSL Behring, Grifols, and Baxter UK; is a coordinating editor of the Cochrane Collaboration Neuromuscular Disease Group; and provides clinical leadership and advice to the National Health Service provided National Hospital for Neurology and Neuroimmunology and CSF Laboratory. VEGF assays are performed by this laboratory for clinical diagnostic purposes. Approximately 1,000 assays are performed each year, constituting $<2 \%$ of laboratory activity per annum; he received research support from the UCLH NHS Foundation. Full disclosure form information provided by the authors is available with the full text of this article at Neurology.org/NN.

Received March 2, 2018. Accepted in final form June 29, 2018.

\section{References}

1. Dispenzieri A. POEMS syndrome: 2017 update on diagnosis, risk-stratification, and management. Am J Hematol 2014;89:214-223.

2. Watanabe O, Arimura K, Kitajima I, Osame M, Maruyama I. Greatly raised vascular endothelial growth factor (VEGF) in POEMS syndrome. Lancet 1996;347:702.

3. Watanabe O, Maruyama I, Arimura K, et al. Overproduction of vascular endothelial growth factor/vascular permeability factor is causative in Crow-Fukase (POEMS) syndrome. Muscle Nerve 1998;21:1390-1397.

4. D'Souza A, Hayman SR, Buadi F, et al. The utility of plasma vascular endothelial growth factor levels in the diagnosis and follow-up of patients with POEMS syndrome. Blood 2011;118:4663-4665.

5. Dispenzieri A. POEMS syndrome. Blood Rev 2007;21:285-299. 
6. D'Souza A, Lacy M, Gertz M, et al. Long-term outcomes after autologous stem cell transplantation for patients with POEMS syndrome (osteosclerotic myeloma) a single-center experience. Blood 2012;120:56-62.

7. Ferrara N. Vascular endothelial growth factor: basic science and clinical progress. Endocr Rev 2004;25:581-611.

8. Hetland ML, Christensen IJ, Lottenburger T, et al. Circulating VEGF as a biological marker in patients with rheumatoid arthritis? Preanalytical and biological variability in healthy persons and in patients. Dis Markers 2008;24:1-10.

9. Tokashiki T, Hashiguchi T, Arimura K, Eiraku N, Maruyama I, Osame M. Predictive value of serial platelet count and VEGF determination for the management of DIC in the Crow-Fukase (POEMS) syndrome. Intern Med 2003;42:1240-1243.

10. Nobile-Orazio E, Terenghi F, Giannotta C, Gallia F, Nozza A. Serum VEGF levels in POEMS syndrome and in immune-mediated neuropathies. Neurology 2009;72: 1024-1026.

11. Stankowski-drengler T, Gertz MA, Katzmann JA, et al. Serum immunoglobulin free light chain measurements and heavy chain isotype usage provide insight into disease biology in patients with POEMS syndrome. Am J Hematol 2011;85:431-434.

12. Aguayo A, Kantarjian HM, Estey EH, et al. Plasma vascular endothelial growth factor levels have prognostic significance in patients with acute myeloid leukemia but not in patients with myelodysplastic syndromes. Cancer 2002;95:1923-1930.

13. Avramis IA, Panosyan EH, Dorey F, Holcenberg JS, Avramis VI. Correlation between high vascular endothelial growth factor-A serum levels and treatment outcome in patients with standard-risk acute lymphoblastic leukemia: a report from Children's Oncology Group Study CCG-1962. Clin Cancer Res 2006;12:6978-6984.

14. Paesler J, Gehrke I, Poll-Wolbeck SJ, Kreuzer KA. Targeting the vascular endothelial growth factor in hematologic malignancies. Eur J Haematol 2012;89:373-384.

15. Sezer O, Jakob C, Eucker J, et al. Serum levels of the angiogenic cytokines basic fibroblast growth factor (bFGF), vascular endothelial growth factor (VEGF) and hepatocyte growth factor (HGF) in multiple myeloma. Eur J Haematol 2001;66: 83-88.

16. Anagnostopoulos A, Eleftherakis-Papaiakovou V, Kastritis E, et al. Serum concentrations of angiogenic cytokines in Waldenstrom macroglobulinaemia: the ration of angiopoietin-1 to angiopoietin-2 and angiogenin correlate with disease severity. $\mathrm{Br} \mathrm{J}$ Haematol 2007; 137:560-568.

17. Ferrara N, Gerber HP, LeCouter J. The biology of VEGF and its receptors. Nat Med 2003;9:669-676.

18. Dunst J, Becker A, Lautenschlager C, et al. Anemia and elevated systemic levels of vascular endothelial growth factor (VEGF). Strahlenther Onkol 2002;178:436-441.

19. Porta C, Imarisio I, De Amici M, et al. Pro-neoangiogenic cytokines (VEGF and bFGF) and anemia in solid tumor patients. Oncol Rep 2005;13:689-695.

20. Eckard J, Dai J, Wu J, et al. Effects of cellular iron deficiency on the formation of vascular endothelial growth factor and angiogenesis: iron deficiency and angiogenesis. Cancer Cell Int 2010;10:28.

21. Jian J, Yang Q, Dai J, et al. Effects of iron deficiency and iron overload on angiogenesis and oxidative stress: a potential dual role for iron in breast cancer. Free Radic Biol Med 2011;50:841-847.

22. Pavlisa G, Kusec V, Kolonic SO, Markovic AS, Jaksic B. Serum levels of VEGF and bFGF in hypoxic patients with exacerbated COPD. Eur Cytokine Netw 2010;21:92-98.

23. Valipour A, Schreder M, Wolzt M, et al. Circulating vascular endothelial growth factor and systemic inflammatory markers in patients with stable and exacerbated chronic obstructive pulmonary disease. Clin Sci (Lond) 2008;115:225-232.
24. Lavie L, Kraiczi H, Hefetz A, et al. Plasma vascular endothelial growth factor in sleep apnea syndrome: effects of nasal continuous positive air pressure treatment. Am J Respir Crit Care Med 2002;165:1624-1628.

25. Teramoto $\mathrm{S}$, Kume $\mathrm{H}$, Yamamoto $\mathrm{H}$, et al. Effects of oxygen administration on the circulating vascular endothelial growth factor (VEGF) levels in patients with obstructive sleep apnea syndrome. Intern Med 2003;42:681-685.

26. Sakai K, Komai K, Yanase D, Yamada M. Plasma VEGF as a marker for the diagnosis and treatment of vasculitic neuropathy. J Neurol Neurosurg Psychiatry 2005;76:296.

27. Carvalho JF, Blank M, Shoenfeld Y. Vascular endothelial growth factor (VEGF) in autoimmune diseases. J Clin Immunol 2007;27:246-256.

28. Sekiya M, Ohwada A, Miura K, Takahashi S, Fukuchi Y. Serum vascular endothelial growth factor as a possible prognostic indicator in sarcoidosis. Lung 2003;181: 259-265

29. Taylor PC. Serum vascular markers and vascular imaging in assessment of rheumatoid arthritis disease activity and response to therapy. Rheumatology (Oxford) 2005;44: 721-728.

30. Choi IS, Byeon JH, Yoo Y, Lee KC, Choung JT. Increased serum interleukin-5 and vascular endothelial growth factor in children with acute mycoplasma pneumonia and wheeze. Pediatr Pulmonol 2009;44:423-428.

31. Urbaniak A, Zieba M, Zwolinska A, et al. Comparison of local and systemic inflammatory markers in patients with community-acquired pneumonia and pneumoni coexisting with lung cancer [in Polish]. Pneumonol Alergol Pol 2011;79:90-98.

32. Distler JH, Hirth A, Kurowska-Stolarska M, Gay RE, Gay S, Distler O. Angiogenic and angiostatic factors in the molecular control of angiogenesis. QJ Nucl Med 2003;47: 149-161.

33. Bachtiary B, Selzer E, Knocke TH, Potter R, Obermair A. Serum VEGF levels in patients undergoing primary radiotherapy for cervical cancer: impact on progressionfree survival. Cancer Lett 2002;179:197-203.

34. Duque JL, Loughlin KR, Adam RM, Kantoff P, Mazzucchi E, Freeman MR. Measurement of plasma levels of vascular endothelial growth factor in prostate cancer patients: relationship with clinical stage, Gleason score, prostate volume, and serum prostate-specific antigen. Clinics (Sao Paulo) 2006;61:401-408.

35. Jablonska E, Puzewska W, Grabowska Z, Jablonski J, Talarek L. VEGF, IL-18 and NO production by neutrophils and their serum levels in patients with oral cavity cancer. Cytokine 2005;30:93-99.

36. Min BS, Kim NK, Jeong HC, Chung HC. High levels of serum VEGF and TIMP-1 are correlated with colon cancer liver metastasis and intrahepatic recurrence after liver resection. Oncol Lett 2012;4:123-130.

37. Obermair A, Hefler L, Nather A, Preyer O, Kaider A. Correlation of the serum concentration of vascular endothelial growth factor (VEGF) and hemoglobin levels in patients with epithelial ovarian cancer. Ann Oncol 1999;10:998.

38. Salgado R, Vermeulen PB, Benoy I, et al. Platelet number and interleukin-6 correlate with VEGF but not with bFGF serum levels of advanced cancer patients. Br J Cancer 1999;80:892-897.

39. Salven P, Perhoniemi V, Tykka H, Maenpaa H, Joensuu H. Serum VEGF levels in women with a benign breast tumor or breast cancer. Breast Cancer Res Treat 1999;53:161-166.

40. Salven P, Ruotsalainen T, Mattson K, Joensuu H. High pre-treatment serum level of vascular endothelial growth factor (VEGF) is associated with poor outcome in smallcell lung cancer. Int J Cancer 1998;79:144-146.

41. Scarlato M, Previtali SC, Carpo M, et al. Polyneuropathy in POEMS syndrome: role of angiogenic factors in the pathogenesis. Brain 2005;128:1911-1920. 


\section{Neurology \\ Neuroimmunology \& Neuroinflammation}

\section{Raised VEGF: High sensitivity and specificity in the diagnosis of POEMS syndrome Morgane Pihan, Stephen Keddie, Shirley D'Sa, et al. \\ Neurol Neuroimmunol Neuroinflamm 2018;5; \\ DOI 10.1212/NXI.0000000000000486}

This information is current as of August 15, 2018

\section{Updated Information \& Services}

References

Citations

Subspecialty Collections

Permissions \& Licensing

Reprints including high resolution figures, can be found at: http://nn.neurology.org/content/5/5/e486.full.html

This article cites 41 articles, 4 of which you can access for free at: http://nn.neurology.org/content/5/5/e486.full.html\#\#ref-list-1

This article has been cited by 2 HighWire-hosted articles: http://nn.neurology.org/content/5/5/e486.full.html\#\#otherarticles

This article, along with others on similar topics, appears in the following collection(s):

Diagnostic test assessment

http://nn.neurology.org//cgi/collection/diagnostic_test_assessment_ Hematologic

http://nn.neurology.org//cgi/collection/hematologic

Peripheral neuropathy

http://nn.neurology.org//cgi/collection/peripheral_neuropathy

Information about reproducing this article in parts (figures,tables) or in its entirety can be found online at:

http://nn.neurology.org/misc/about.xhtml\#permissions

Information about ordering reprints can be found online: http://nn.neurology.org/misc/addir.xhtml\#reprintsus

Neurol Neuroimmunol Neuroinflamm is an official journal of the American Academy of Neurology.

Published since April 2014, it is an open-access, online-only, continuous publication journal. Copyright

Copyright (C) 2018 The Author(s). Published by Wolters Kluwer Health, Inc. on behalf of the American

Academy of Neurology.. All rights reserved. Online ISSN: 2332-7812.

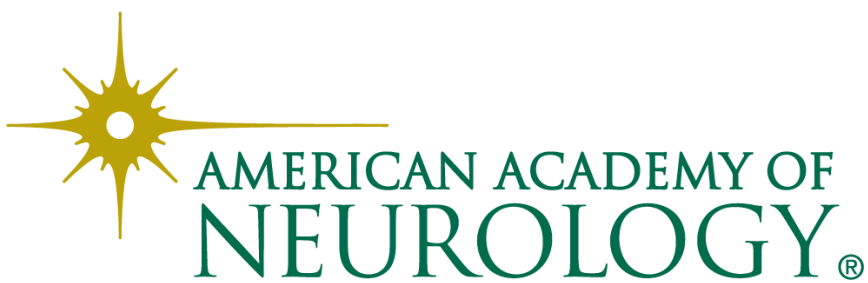

